\title{
Poly(ADP) ribose polymerase-1 ablation alters eicosanoid and docosanoid signaling and metabolism in a murine model of contact hypersensitivity
}

\author{
BORBÁLA KISS ${ }^{1 *}$, MAGDOLNA SZÁNTÓ ${ }^{2,3 *}$, MÓNIKA SZKLENÁR ${ }^{4}$, ATTILA BRUNYÁNSZKI $^{2}$, \\ TAMÁS MAROSVÖLGYI ${ }^{5}$, ESZTER SÁROSI ${ }^{5}$, ÉVA REMENYIK ${ }^{1}$, \\ PÁL GERGELY ${ }^{2}$, LÁSZLÓ VIRÁG ${ }^{2,3}$, TAMÁS DECSI ${ }^{5}$, RALPH RÜHL ${ }^{6}$ and PETER BAI ${ }^{2,7,8}$ \\ Departments of ${ }^{1}$ Dermatology and ${ }^{2}$ Medical Chemistry, University of Debrecen, H-4032 Debrecen; \\ ${ }^{3}$ MTA-DE Cell Biology and Signaling Research Group of the Hungarian Academy of Sciences, H-4032 Debrecen; \\ ${ }^{4}$ Paprika Bioanalytics Bt., H-4032 Debrecen; ${ }^{5}$ Department of Pediatrics, University of Pécs, H-7623 Pécs; \\ ${ }^{6}$ Department of Biochemistry and Molecular Biology and ${ }^{7}$ Research Center for Molecular Medicine, University of Debrecen, \\ H-4032 Debrecen; ${ }^{8}$ MTA-DE Lendület Laboratory of the Cellular Metabolism Research Group, \\ H-4032 Debrecen, Hungary
}

Received January 22, 2014; Accepted September 24, 2014

DOI: $10.3892 / \mathrm{mmr} .2014 .3044$

\begin{abstract}
Poly(ADP-ribose) polymerase (PARP)-1 is a pro-inflammatory protein. The inhibition of PARP-1 reduces the activity of numerous pro-inflammatory transcription factors, which results in the reduced production of pro-inflammatory cytokines, chemokines, matrix metalloproteinases and inducible nitric oxide synthase, culminating in reduced inflammation of the skin and other organs. The aim of the present study was to investigate the effects of the deletion of PARP-1 expression on polyunsaturated fatty acids (PUFA), and PUFA metabolite composition, in mice under control conditions or
\end{abstract}

Correspondence to: Dr Peter Bai, Department of Medical Chemistry, University of Debrecen, Nagyerdei krt 98. Pf. 7. H-4032 Debrecen, Hungary

E-mail: baip@med.unideb.hu

Dr Ralph Rühl, Department of Biochemistry and Molecular Biology, University of Debrecen, Nagyerdei krt 98, H-4032 Debrecen, Hungary

E-mail: ralphruehl@web.de

*Contributed equally

Abbreviations: AA, arachidonic acid; CHS, contact hypersensitivity; COX, cyclooxygenase; DAPI, 4,6-diamidino-2-phenylindole; DHA, docosahexaenoic acid; DHGLA, dihomo- $\gamma$-linoleic acid; EPA, eicosapentaenoic acid; FABP, fatty acid binding proteins; MET, metabolite; OXA, oxazolone; PARP, poly(ADP-ribose) polymerase; PUFAs, polyunsaturated fatty acids; Pg, prostaglandin

Key words: PARP-1, PUFA, contact hypersensitivity, oxazolone, DHA, EPA, FABP7, lipidomics, inflammation, cyclooxygenase-2 undergoing an oxazolone (OXA)-induced contact hypersensitivity reaction (CHS). CHS was elicited using OXA in both the PARP $-1^{+/+}$and PARP $-1^{-/-}$mice, and the concentration of PUFAs and PUFA metabolites in the diseased skin were assessed using lipidomics experiments. The levels of docosahexaenoic acid (DHA) and eicosapentaenoic acid (EPA) were shown to be increased in the PARP-1 $1^{-/}$mice, as compared with the control, unsensitized PARP-1 ${ }^{+/+}$mice. In addition, higher expression levels of fatty acid binding protein 7 (FABP7) were detected in the PARP-1 $1^{-/}$mice. FABP7 is considered to be a specific carrier of DHA and EPA. Furthermore, the levels of the metabolites of DHA and EPA (considered mainly as anti-inflammatory or pro-resolving factors) were higher, as compared with the metabolites of arachidonic acid (considered mainly pro-inflammatory), both in the unsensitized control and OXA-sensitized PARP-1 ${ }^{-/-}$mice. The results of the present study suggest that the genetic deletion of PARP-1 may affect the PUFA-homeostasis of the skin, resulting in an anti-inflammatory milieu, including increased DHA and EPA levels, and DHA and EPA metabolite levels. This may be an important component of the anti-inflammatory action of PARP-1 inhibition.

\section{Introduction}

Poly(ADP-ribose) polymerases (PARPs) comprise a superfamily containing 17 members in humans (1). Numerous PARP-1, -2, -14 and tankyrases have previously been shown to mediate inflammatory reactions (2-6), and PARP-1 has been associated with inflammatory or oxidative stress-related skin pathologies (7-9). PARP-1 is an important pro-inflammatory mediator with important roles in the maturation of immune cells (10-12), activation of pattern recognition receptors (13-19), activation of pro-inflammatory transcription factors (6), and reactive species (20). 
The contact hypersensitivity (CHS) reaction is a T-cell mediated, delayed type hypersensitivity reaction that is elicited by low molecular weight molecules, such as oxazolone (OXA) $(21,22)$. Upon sensitization, infiltrating cells and keratinocytes produce free radicals, pro-inflammatory cytokines, adhesion molecules and matrix metalloproteinases in a PARP-1 and nuclear factor- $\kappa \mathrm{B}$-dependent manner, resulting in the induction of inflammation (6,7,20,23-25). PARP-1 expression inhibition, or knock-out, has been shown to reduce the severity of experimental CHS $(7,26)$. The present study aimed to characterize whether alterations of eicosanoid and docosanoid metabolism and signaling may contribute to the anti-inflammatory effects of PARP-1 expression ablation.

\section{Materials and methods}

Materials. OXA and the other chemicals used in the present study were obtained from Sigma-Aldrich (St. Louis, MO, USA), unless stated otherwise.

Animal experiments. All of the experiments performed on mice were approved by the Local Ethical Committee (9/2008/DE MÁB) and were performed according to EU and national guidelines. PARP-1 ${ }^{+/+}$and PARP-1/- mice, derived from heterozygote-to-heterozygote breeding, were kept in the animal facility of the Life Science Building, University of Debrecen (Debrecen, Hungary) (27). A CHS model was constructed as described by previous methods (7). Briefly, the mice were randomized into four groups: PARP $-1^{+/+}$vehicle sensitized (control), PARP-1 ${ }^{-/-}$vehicle sensitized (control), PARP- ${ }^{+/+} \mathrm{OXA}$ sensitized (CHS) and PARP-1 ${ }^{-/}$OXA sensitized (CHS) groups $(n=5 / 5 / 6 / 5)$. Sensitization was carried out on the shaved abdominal wall skin of the mice, with an administration of $100 \mu 12 \%$ OXA solubilised in acetone : olive oil (4:1; CHS groups), or $100 \mu \mathrm{l}$ vehicle of acetone:olive oil (4:1; control groups). Seven days after sensitization, all of the mice were challenged with $100 \mu 10.5 \%$ OXA, which was applied to the epidermis of the shaved back. After $24 \mathrm{~h}$, the mice were sacrificed and the skin was harvested.

Histology and microscopy. Hematoxylin-eosin staining and immunohistochemistry was performed on paraffin-fixed $7 \mu \mathrm{m}$ tissue sections, as described previously (7).

High performance liquid chromatography-electrospray tandem mass spectrometry (HPLC-ESI-MS-MS) analysis. The procedure for the HPLC-ESI-MS-MS analysis The analysis of free fatty acids, eicosanoids and docosanoids in the skin was performed, according to previously described methods (28), with minor modifications.

Sample preparation. The whole analytical sample preparation procedure used was based on an established method used for retinoid quantification (29). Briefly, $50 \mathrm{mg}$ skin biopsy, was treated with $150 \mu \mathrm{l}$ acetonitrile and the skin samples were cut into small pieces with scissors, on ice. If less than $50 \mathrm{mg}$ of skin biopsy was present, water was added to yield $50 \mathrm{mg}$ of sample weight. The mixtures were agitated for $3 \mathrm{~min}$, and the precipitated protein was centrifuged at $16060 \mathrm{~g}(13,000 \mathrm{rpm})$, $4^{\circ} \mathrm{C}$ for $6 \mathrm{~min}$. A total of $130 \mu \mathrm{l}$ resulting supernatant was mixed with $10 \mu \mathrm{l}$ isotope supernatant mix and evaporated in
Eppendorf reaction vials (Eppendorf, Hamburg, Germany) using an Eppendorf concentrator at $30^{\circ} \mathrm{C}$ for $\sim 60 \mathrm{~min}$, until the sample volume was $\sim 10 \mu$ l. The Eppendorf concentrator was vented with argon, in order to prevent degradation of eicosanoids and docosanoids. The dried extract was resuspended in $25 \mu \mathrm{l}$ HPLC solvent A [64.3\% water (Chromasolv Plus; Sigma-Aldrich, Budapest, Hungary), 35.5\% acetonitrile (Merck KGaA, Darmstadt, Germany) and $0.2 \%$ formic acid (Sigma-Aldrich, Hungary)] to yield $35 \mu \mathrm{l}$. The sample was then vortexed (15 sec), agitated (3 min) and transferred into micro injection inserts vials (Waters, Budapest, Hungary). The glass vials containing $35 \mu \mathrm{l}$ extract were transferred into brown screw top vials with PTFE/silicone septa (Waters), and placed into the pre-cooled $\left(15^{\circ} \mathrm{C}\right)$ autosampler of the Waters $2695 \mathrm{XE}$ separation module.

Chromatographic system. The HPLC system consisted of a Waters 2695XE separation module (Waters) including a gradient pump, autosampler, degasser and a heated column compartment. A MS-MS detector, with an ESI ionizing option, (Micromass Quattro Ultima PT; Waters, Elstree, UK; a gift from Biosystems Int., France) was used as the detector. The system was controlled using MassLynx software (Waters).

HPLC conditions. The eluents were degassed in the Waters 2695XE separation module prior to mixing, and passed through an in-line filter (1-2 $\mu \mathrm{m}$; Knauer, Berlin, Germany) before reaching the analytical column (LiChroCART, 125x 2 mm; Superspher 100, RP-18, endcapped; Merck KgaA), which was embedded in the column compartment. A multilinear gradient was formed from solvent A (as mentioned previously) and solvent B (methanol; Merck KGaA). The gradient consisted of the following steps: $0.0 \mathrm{~min} 20 \% \mathrm{~B}, 3.0 \mathrm{~min} 20 \% \mathrm{~B}, 5.0 \mathrm{~min}$ $60 \% \mathrm{~B}, 15.0 \mathrm{~min} 100 \% \mathrm{~B}, 15.9 \mathrm{~min} 100 \% \mathrm{~B}$ and $16.0 \mathrm{~min} 5 \%$ B. The flow rate was adjusted to $0.4 \mathrm{ml} / \mathrm{min}$ and the column was heated to $40^{\circ} \mathrm{C}$. From the same biological extract, $10 \mu \mathrm{l}$ was used for each HPLC analysis. This step was performed twice, using the same HPLC conditions, and two different MS-MS analysis options were conducted for better resolution and quantification of the various analytes.

MS options. The Micromass Quattro Ultima PT was controlled using MassLynx software. Argon was used with an inlet pressure of 0.8 bar. ESI (electro spray ionization source; Waters) was vented by nitrogen, which was continuously produced by a nitrogen generator (Peak Scientific NM30 Nitrogen generator; Peak Scientific, Billerica, MA, USA) including a compressor (Waters), the inlet flow was set at $3.6 \times 10^{-3}$ mbar.

Multiple reaction monitoring settings. ESI, with a negative ESI-setting, was performed with the HPLC eluent, following the ion source temperature of $85^{\circ} \mathrm{C}$. The desolvation gas flow was $780 \mathrm{l} / \mathrm{h}$, the desolvation temperature was $400^{\circ} \mathrm{C}$, the cone gas flow was $10 \mathrm{l} / \mathrm{h}$, the capillary current was $3 \mu \mathrm{A}$, and the cone voltage was $50 \mathrm{~V}$. The aperture voltage was set at $0 \mathrm{~V}$ and the range-finder lens voltage was set at $35 \mathrm{~V}$ (for one) and $0.2 \mathrm{~V}$ (for two). The analyzer settings were LM1 resolution, 14.5; HM1 resolution, 14.5; ion energy, 10.7; entrance, -1; collision, 0 (collision parameters were set for each substance at the MS-method parameters); exit, 2; LM2 resolution, 14.5; HM2 resolution, 14.5; ion energy, 25.0 and multiplier energy, $650 \mathrm{~V}$. 
Table I. Oligonucleotides used in quantitative polymerase chain reactions.

\begin{tabular}{ll}
\hline Gene & \multicolumn{1}{c}{ Sequence } \\
\hline Mouse 36B4 & 5'-AGA TTC GGG ATA TGC TGT TGG-3' \\
Mouse GAPDH & 5'-AAA GCC TGG AAG AAG GAG GTC-3' \\
Mouse cyclophyllin & 5'-CAA GGT CAT CCA TGA CAA CTT TG-3' \\
Mouse FABP7 & 5'-GGC CAT CCA CAG TCT TCT GG-3' \\
Mouse COX2 & 5'-TGG AGA GCA CCA AGA CAG ACA-3' \\
Human 36B4 & 5'-TGC CGG AGT CGA CAA TGA T-3' \\
Human actin & 5'-GAG TAC ATG AAA GCT CTG GGC-3' \\
Human cyclophyllin & 5'-AAC CGA ACC ACA GAC TTA CAGT T-3' \\
Human PARP-1 & 5'-GTG AAG GGAAAT AAG GAG CTT CC-3' \\
& 5'-GTG ATT TAA GTC CAC TCC ATG GC-3' \\
& 5'-CCA TTG AAA TCC TGA GTG ATG TG-3' \\
& 5'-GTC GAA CAC CTG CTG GAT GAC-3' \\
& 5'-GAC CCA GAT CAT GTT TGA GAC C-3' \\
& 5'-CAT CAC GAT GCC AGT GGT AC-3' \\
& 5'-GTC TCC TTT GAG CTG TTT GCA GAC-3' \\
& 5'-CTT GCC ACC AGT GCC ATT ATG-3'
\end{tabular}

Multiple reaction monitoring settings for polyunsaturated fatty acids (PUFA), eicosanoids/docosanoids semi-quantification. The following methods were used, as described in a previous study (27): Method A from 0.0-9.0 min for $\mathrm{PGF}_{2} 349.0$-> 192.7, collision energy $22 \mathrm{eV}$; PD1 and PD1 isomers, like PDX, 359.0 -> 153.3, collision energy 17 eV; TXB $_{2} 369.0$-> 195.0, collision energy $13 \mathrm{eV} ; \mathrm{PGE}_{3} 349.0$-> 233.0, collision energy $17 \mathrm{eV}$; $\mathrm{LXA}_{4} / \mathrm{LXB}_{4} 351.0 \rightarrow 115.0$, collision energy $12 \mathrm{eV} ; 8 \mathrm{PGF}_{3}$ $351.0 \rightarrow 193.0$, collision energy $22 \mathrm{eV} ; 20-\mathrm{COOH}_{-} \mathrm{LTB}_{4}$ 365.0 -> 195.0, collision energy $13 \mathrm{eV}$; RvD1, RvD2 375.0 -> 141.3, collision energy $13 \mathrm{eV}$; RvE1 375.1 -> 141.3, collision energy 13 eV. From 9.0-12.5 min for 13-HODE 294.7 -> 170.7, collision energy $16 \mathrm{eV}$; 9-HODE 294.7 -> 194.7, collision energy $16 \mathrm{eV}$; 5-HEPE 317.0 -> 115.0, collision energy $17 \mathrm{eV}$; 12-HEPE 317.0 -> 179.0, collision energy $17 \mathrm{eV}$; 15-HEPE 317.0 -> 219.0, collision energy $17 \mathrm{eV}, \mathrm{LTC}_{4} 623.9$-> 272.0, collision energy $14 \mathrm{eV}$. From $12.5-16.0 \mathrm{~min}$ for LA 279.3 -> 279.0, collision energy $10 \mathrm{eV}$; 8-HEPE 317.0 -> 255.0, collision energy $17 \mathrm{eV}$; 18-HEPE 317.0 -> 259.0, collision energy $17 \mathrm{eV}$; 5-oxoETE, 12-oxoETE and 15-oxoETE 317.0 $\rightarrow$ 273.0, collision energy $17 \mathrm{eV}$; 20-HETE $319.0->245.0$, collision energy $10 \mathrm{eV} ; \mathrm{LTC}_{4} 623.9$-> 272.0, collision energy $14 \mathrm{eV} ; \mathrm{LTE}_{4} 438.0$-> 333.0, collision energy $13 \mathrm{eV}$. From 12.5-16.0 min for LA 279.3 -> 59.2, collision energy $25 \mathrm{eV}$; EPA 301.0 -> 203.2, collision energy $12 \mathrm{eV}$; AA 303.0 -> 259.3, collision energy $14 \mathrm{eV}$; DHA 327.1 -> 29.3, collision energy $14 \mathrm{eV}$.

Method B (27) from 0.0-9.8 min for $\mathrm{PGE}_{2}, \mathrm{~d} 15 \mathrm{~d} 12 \mathrm{PGD}_{2}$, $\mathrm{PGD}_{2}, \mathrm{~d} 15 \mathrm{~d} 12 \mathrm{PGJ}_{2}$ and $\mathrm{PGJ}_{2} 315.0 \rightarrow 271.3$, collision energy $13 \mathrm{eV} ; \mathrm{LTB}_{5} 333.0$-> 195.0, collision energy $13 \mathrm{eV} ; \mathrm{LTB}_{4} 335.0$ -> 195.0, collision energy $13 \mathrm{eV}$; $\mathrm{RvE}_{1} 349.1$-> 195.3, collision energy $13 \mathrm{eV} ; \mathrm{HXA}_{3}, \mathrm{HXB}_{3}$ and 20-COOH-AA 335.0 -> 273.3, collision energy $13 \mathrm{eV}$; LXA $349.0 \rightarrow 115.0$, collision energy $12 \mathrm{eV}$; 20-OH-LTB 351.0 -> 195.0, collision energy $13 \mathrm{eV}$;
MaR 359.0 -> 250.0, collision energy $13 \mathrm{eV}$. From 9.8-12.5 min for 5-HETE 318.7 -> 115.0, collision energy $14 \mathrm{eV}$; 8-HETE 319.0 -> 155.0, collision energy $14 \mathrm{eV}$; 11-HETE 319.0 -> 167.0, collision energy $14 \mathrm{eV}$; 12-HETE 319.0 -> 179.0, collision energy 14 eV; 15-HETE 319.0 -> 218.9, collision energy $11 \mathrm{eV}$; 4-HDHA $343.0->$ 101.0, collision energy $10 \mathrm{eV}$; 10-HDHA 343.0 -> 181.0, collision energy $10 \mathrm{eV}$; 14-HDHA 343.0 -> 205.0, collision energy $10 \mathrm{eV}$; 17-HDHA $343.0->245.0$, collision energy $14 \mathrm{eV}, 20$-HDHA 343.0 -> 285.0, collision energy $10 \mathrm{eV}$; 13-oxoODE 293.0 -> 249.0, collision energy $17 \mathrm{eV}$. And from 12.5-16.0 min for LA 279.3 -> 59.2, collision energy $25 \mathrm{eV}$; EPA 301.0 -> 203.2, collision energy $12 \mathrm{eV}$; AA $303.0->$ 259.3, collision energy $11 \mathrm{eV}$; and DHA 327.1 -> 229.3, collision energy $14 \mathrm{eV}$.

Standard solutions. Stock solutions of the PUFAs, eicosanoids and docosanoids were prepared by dissolving the solutions in methanol to yield a final concentration of $10 \mu \mathrm{g} / \mathrm{ml}$. The solutions were obtained from Cayman-Chemicals (Tallinn, Estonia), BioMol International (Kastel-Med KFT, Budapest, Hungary), Sigma-Aldrich(Hungary),LarodanLipids(Malmö,Sweden)and Dr. Charles Serhan (Harvard, MA, USA). All stock solutions were stored in the dark, at $-80^{\circ} \mathrm{C}$ until further use. The reference PUFAs, eicosanoids and docosanoids (BioMol International/ Enzo Life Sciences, Farmingdale, NY, USA) were used for assay validation.

Quantification. Individual eicosanoids and docosanoids were quantified based on the determination of the area under the curve (AUC), which was compared with the AUC of the standard compounds. To ensure optimal extraction, isotope-labelled standard compounds were used. This analytical procedure was previously established for liquids and tissue analysis (28). 

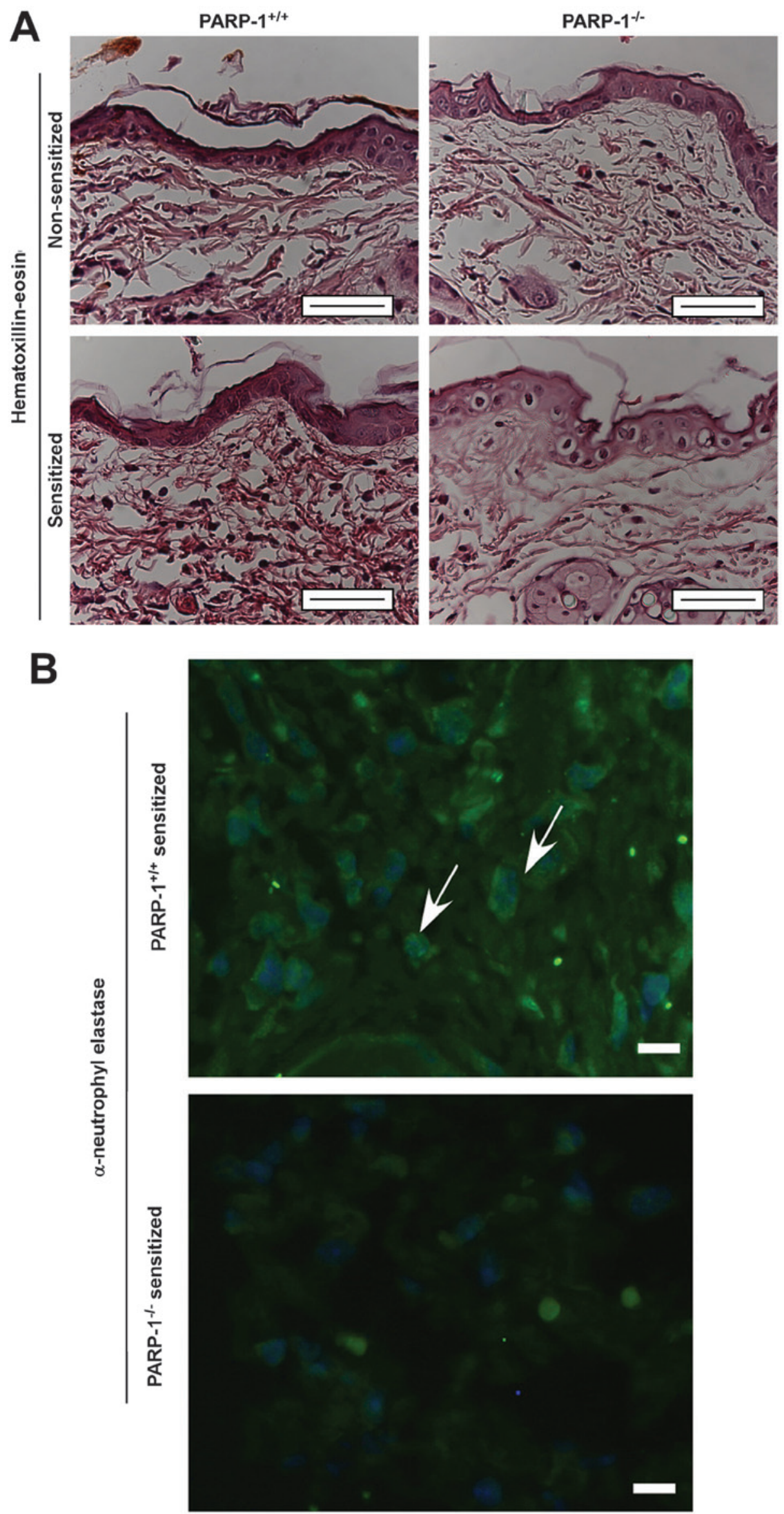

Figure 1. Deletion of poly(ADP-ribose) polymerase-1 (PARP-1) suppresses neutrophil migration. Formalin-fixed, paraffin-embedded tissue sections were stained with (A) hematoxylin and eosin and (B) DAPI counterstain (blue) and an anti-neutrophil elastase antibody (green) to detect neutrophil elastase. The arrows indicate the neutrophil elastase-positive cells. The neutrophil elastase staining was observed to be cytoplasmic. Scale bars: A, $30 \mu \mathrm{m}$; B, $10 \mu \mathrm{m}$. 

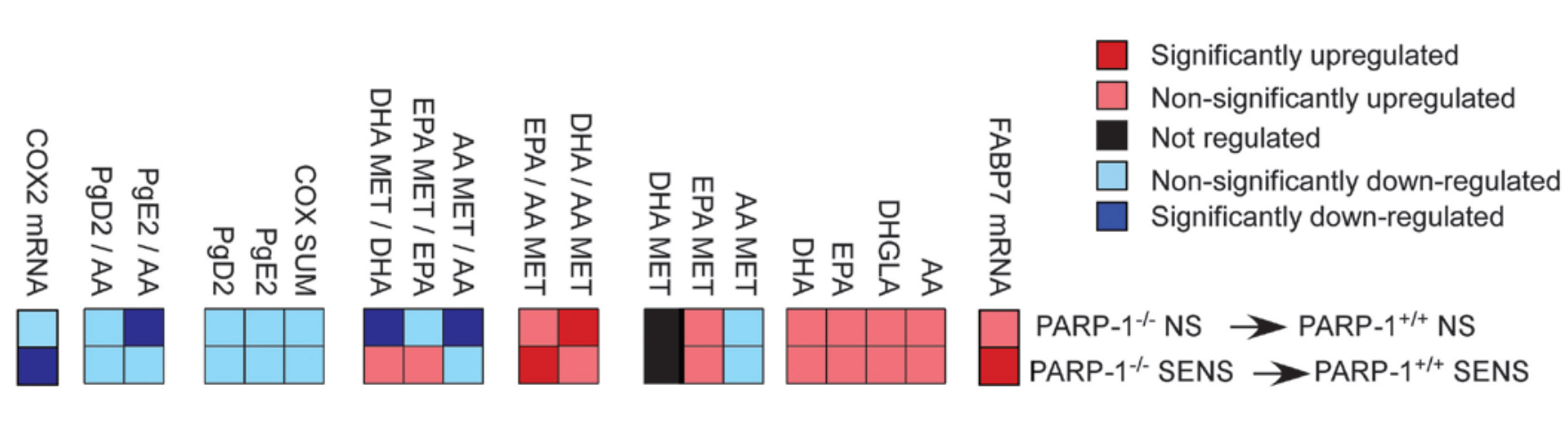

Figure 2. Eicosanoid and docosanoid signaling alterations in the skin of poly(ADP-ribose) polymerase-1 (PARP-1) ${ }^{-/-}$mice, due to enhanced fatty acid binding protein (FABP)7 expression. The FABP7 mRNA expression levels, and eicosanoid and docosanoid concentrations, were determined in the skin of control (PARP- $1^{+/+}$NS), OXA-sensitized PARP- $1^{+/+}\left(\right.$PARP- $1^{+/+}$SENS) and respective PARP- $1^{-/-}$mice $(\mathrm{n}=5 / 5 / 6 / 5)$. AA, arachidonic acid; DHGLA, dihomo-gammalinoleic acid; EPA, eicosapentaenoic acid; DHA, docosahexaenoic acid; MET, metabolites; COX, cyclooxygenase; COX2, cyclooxygenase 2; Pg, prostaglandin.

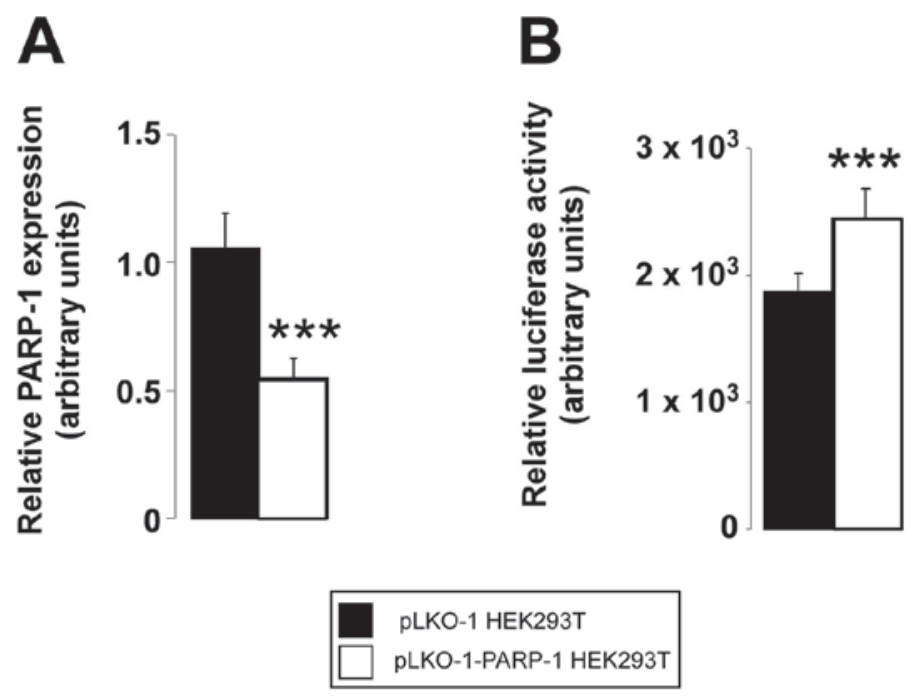

Figure 3. Reduction in the expression levels of poly(ADP-ribose) polymerase-1 (PARP-1) in response to specific small hairpin (sh)RNA treatment of HEK293T human embryonic kidney cells. PARP-shRNA also induced the activity of the fatty acid binding protein (FABP)7 promoter. (A) PARP-1 expression levels were reduced in HEK293T cells using a construct coding an shRNA against PARP-1 (shPARP-1), as determined by quantitative polymerase chain reaction ( $\mathrm{n}=3 / 3$ ). (B) Promoter activity of FABP7 was determined in luciferase reporter assays in control (scPARP-1 HEK2935T) and shPARP-1 HEK293T cells ( $\mathrm{n}=3 / 3$ ). $* \mathrm{P}<0.05$, PARP-1+/+ mice, compared with PARP-1-/- mice; ***P<0.001, scPARP-1, compared with shPARP-1 HEK293T cells.

mRNA preparation, reverse transcription and quantitative polymerase chain reaction ( $q P C R)$. Total RNA extraction, reverse transcription, and a subsequent qPCR, were performed as described previously (7). The primers used are listed in Table I.

Establishment of a PARP-1 knockdown HEK293T cell line and luciferase reporter assays. HEK293T human embryonic kidney cells (American Type Culture Collection, Manassas, VA, USA) were maintained in Dulbecco's modified Eagle's medium, supplemented with $4.5 \mathrm{~g} / 1$ glucose, $10 \%$ fetal calf serum, $2.5 \mu \mathrm{g} / \mathrm{ml}$ puromycin. These cells stable expressing a small hairpin (sh) RNA specifically targeting PARP-1 (pLKO.1-PARP1). pLKO.1 was used in the control cells (Sigma-Aldrich, USA). PARP-1 expression was subsequently suppressed, as confirmed by qPCR.

pLightSwitch_PromFABP7 (Switchgear Genomics, Carlsbad, CA, USA) and pCMV- $\beta$ gal were transfected into the above cell line using jetPEI (PolyPlus Transfections SA, Illkirch, France). Following a $24 \mathrm{~h}$ incubation, the luciferase and $\beta$-galactosidase activities were determined. Luciferase activity was normalized to $\beta$-galactosidase activity.

Statistical analysis. The results of the cell assays are expressed as the means \pm standard deviation, and statistical significance between the groups was determined by Student's t-test. The results of the in vivo studies are expressed as the means, and statistical significance was determined using the Kruskal-Wallis and Mann-Whitney tests. A $\mathrm{P}<0.05$ was considered to indicate a statistically significant difference.

\section{Results}

Neutrophil infiltration and edema at the site of the CHS reaction was observed in the histological examinations; however, 
the levels of these were reduced in the PARP-1 $1^{-/}$mice (Fig. 1A and $\mathrm{B}$ ). This finding indicates that PARP-1 may contribute to inflammation in CHS, therefore PARP-1 $1^{-/}$mice may be protected against CHS. These results are concordant with our previous observations $(7,26,30)$.

The qPCR detected higher mRNA expression levels of fatty acid binding protein (FABP)7 in the PARP-1 $1^{-/}$mice (Fig. 2). The depletion of PARP-1 expression (Fig. 3A) induced the activity of the FABP7 promoter (Fig. 3B), suggesting that PARP-1 may have direct effects on the FABP7 promoter.

Using a lipidomic approach, the composition of eicosanoids and docosanoids in the skin was determined. There was an increase in four of the major PUFAs, which are precursors of eicosanoids and docosanoids: Arachidonic acid (AA; 20:4 n-6), dihomo-gamma-linoleic acid (DHGLA; 20:3 n-6), docosahexaenoic acid (DHA; 22:5 n-3) and eicosapentaenoic acid (EPA; 20:5 n-3). These free fatty acids had increased levels in the skin of the control and OXA-challenged PARP- $1^{-/-}$mice, as compared with their respective PARP-1 $1^{+/+}$ cohorts (Fig. 2).

The levels of AA-metabolites (AA MET), as compared with the mother compound AA (AA MET/AA), as well as EPA MET/EPA and DHA MET/DHA, had lower ratios in the non-sensitized animals, and the n3-PUFAs ratios were increased in the sensitized animals (Fig. 2). The sum of the cyclooxygenase (COX) metabolites (COX SUM), and the levels of COX metabolites prostaglandin E2 (PgE2) and PgD2, as well as the PgE2/AA and PgD2/AA ratios, were all decreased showing reduced synthesis of pro-inflammatory lipid mediators. The levels of anti-inflammatory or pro-resolving n3-PUFA metabolites were mainly increased, resulting from EPA and DHA-metabolism (31) in the PARP-1 $1^{-1}$ animals (Fig. 2). These data are concordant with the decreased expression levels of COX-2 in the PARP-1 ${ }^{-/}$mice(Fig. 2).

\section{Discussion}

In the present study, it was determined that ablation of PARP-1 expression had characteristic effects on skin eicosanoid and docosanoid signaling, and metabolism. The two n-3 PUFAs (DHA and EPA), DHA- and EPA-metabolites, and the ratios of DHA- and EPA-metabolites vs. AA-metabolites were increased, indicating a pro-resolving, anti-inflammatory environment (31) in the PARP- $1^{-/-}$mice, especially following OXA-sensitization. The formation of the pro-inflammatory COX-metabolites was lower in the skin of the PARP-1 ${ }^{-/}$mice, which is concordant with a previously determined anti-inflammatory environment (6). PARP-1 has previously been shown to regulate COX-2 expression (32) which may explain the observed decrease in COX-2 metabolites.

It may be suggested that increased FABP7-levels are responsible for the observed alterations of lipid mediator signaling. FABP7 has preference towards $\mathrm{n}-3$, as compared with n-6, PUFAs (33), this may be a plausible explanation for the accumulation of EPA-derived metabolites, and increased ratios of PUFA-metabolites (DHA/AA MET, EPA/AA MET) in the PARP- $1^{+/+}$mice.

The results of the present study widen the spectrum of altered lipid metabolism upon PARP-1 expression ablation $(34,35)$, by implicating skin eicosanoid and docosanoid metabolism and signaling. A reduction in PARP-1 expression results in a reduced pro-inflammatory and increased pro-resolving or anti-inflammatory environment in the skin. Higher levels of the DHA and EPA metabolites, and reduced COX-pathway metabolites were previously implicated in a reduction of DNFB-induced CHS (36), peritonitis (31), atopy (37) or allergy (38).

These data are concordant with our previous findings, which showed that genetic or pharmacological inhibition of PARP-1 is capable of protecting against CHS $(7,26,39)$. Notably, differences in eicosanoid and docosanoid metabolism and signaling are also present in unchallenged mice and it may be speculated that these minor changes may render ${\text { PARP }-1^{-/}}^{-1}$ mice less susceptible to CHS. Furthermore, the changes to gene expression levels and lipid mediator composition are associated with inflammatory pathologies that are mediated not only by T-helper (Th) 1 or Th 2 cells, but also the newly defined Th17 cells, which have previously been associated with the induction of FABPs in psoriasis (40). In conclusion, alteration in skin eicosanoid and docosanoid metabolism and signaling may modify the barrier function of the skin, wherein the role of PARPs remains relatively unexplored.

\section{Acknowledgements}

The present study was supported by grants from the National Innovation Office (Baross program Seahorse grant; no. TÁMOP-4.2.2. A-11/1/KONV-2012-0025), OTKA (nos. PD83473, K105872, K108308, CNK80709, K104720 and K108308), and the Medical and Health Science Center (Mecenatura no. Mec-8/2011). PB and MS are recipients of the Bolyai fellowship from the Hungarian Academy of Sciences. The authors of the present study would like to acknowledge the technical assistance of Mrs Erzsébet Herbály.

\section{References}

1. Amé JC, Spenlehauer C and de Murcia G: The PARP superfamily. Bioessays 26: 882-893, 2004.

2. Szántó M, Brunyánszki A, Kiss B, et al: Poly(ADP-ribose) polymerase-2: emerging transcriptional roles of a DNA-repair protein. Cell Mol Life Sci 69: 4079-4092, 2012.

3. Mehrotra P, Hollenbeck A, Riley JP, et al: Poly (ADP-ribose) polymerase 14 and its enzyme activity regulates $\mathrm{T}(\mathrm{H}) 2$ differentiation and allergic airway disease. J Allergy Clin Immunol 131: 521-531, 2013.

4. Mehrotra P, Riley JP, Patel R, et al: PARP-14 functions as a transcriptional switch for Stat6-dependent gene activation. J Biol Chem 286: 1767-1776, 2011.

5. Levaot N, Voytyuk O, Dimitriou I, et al: Loss of Tankyrase-mediated destruction of 3BP2 is the underlying pathogenic mechanism of cherubism. Cell 147: 1324-1339, 2011.

6. Bai P and Virág L: Role of poly(ADP-ribose) polymerases in the regulation of inflammatory processes. FEBS Lett 586: 3771-3777, 2012.

7. Brunyánszki A, Hegedus C, Szántó M, et al: Genetic ablation of PARP-1 protects against oxazolone-induced contact hypersensitivity by modulating oxidative stress. J Invest Dermatol 130: 2629-2637, 2010.

8. Virág L, Szabó E, Bakondi E, et al: Nitric oxide-peroxynitrite-poly (ADP-ribose) polymerase pathway in the skin. Exp Dermatol 11: 189-202, 2002.

9. Bakondi E, Gönczi M, Szabó E, et al: Role of intracellular calcium mobilization and cell-density-dependent signaling in oxidative-stress-induced cytotoxicity in $\mathrm{HaCaT}$ keratinocytes. J Invest Dermatol 121: 88-95, 2003. 
10. Selle A, Ullrich O, Harnacke K and Hass R: Retrodifferentiation and rejuvenation of senescent monocytic cells requires PARP-1. Exp Gerontol 42: 554-562, 2007.

11. Aldinucci A, Gerlini G, Fossati S, et al: A key role for poly(ADP-ribose) polymerase-1 activity during human dendritic cell maturation. J Immunol 179: 305-312, 2007.

12. Mocchegiani E, Muzzioli M, Giacconi R, et al: Metallothioneins/PARP-1/IL-6 interplay on natural killer cell activity in elderly: parallelism with nonagenarians and old infected humans. Effect of zinc supply. Mech Ageing Dev 124: 459-468, 2003.

13. Zerfaoui M, Errami Y, Naura AS, et al: Poly(ADP-ribose) polymerase-1 is a determining factor in Crm1-mediated nuclear export and retention of p65 NF-kappa B upon TLR4 stimulation. J Immunol 185: 1894-1902, 2010.

14. Rolli J, Rosenblatt-Velin N, Li J, et al: Bacterial flagellin triggers cardiac innate immune responses and acute contractile dysfunction. PLoS One 5: e12687, 2010.

15. Eaves-Pyles T, Murthy K, Liaudet L, et al: Flagellin, a novel mediator of Salmonella-induced epithelial activation and systemic inflammation: I kappa B alpha degradation, induction of nitric oxide synthase, induction of proinflammatory mediators, and cardiovascular dysfunction. J Immunol 166: 1248-1260, 2001.

16. Liaudet L, Deb A, Pacher P, et al: The Flagellin-TLR5 axis: Therapeutic opportunities. Drug News Perspect 15: 397-409, 2002.

17. Liaudet L, Murthy KG, Mabley JG, et al: Comparison of inflammation, organ damage, and oxidant stress induced by Salmonella enterica serovar Muenchen flagellin and serovar Enteritidis lipopolysaccharide. Infect Immun 70: 192-198, 2002.

18. Liaudet L, Szabó C, Evgenov OV, et al: Flagellin from gram-negative bacteria is a potent mediator of acute pulmonary inflammation in sepsis. Shock 19: 131-137, 2003.

19. Murthy KG, Deb A, Goonesekera S, Szabó C and Salzman AL: Identification of conserved domains in Salmonella muenchen flagellin that are essential for its ability to activate TLR5 and to induce an inflammatory response in vitro. J Biol Chem 279: 5667-5675, 2004.

20. Pacher P, Beckman JS and Liaudet L: Nitric oxide and peroxynitrite in health and disease. Physiol Rev 87: 315-424, 2007.

21. Grabbe S and Schwarz T: Immunoregulatory mechanisms involved in elicitation of allergic contact hypersensitivity. Immunol Today 19: 37-44, 1998 .

22. Grabbe S, Steinert M, Mahnke K, et al: Dissection of antigenic and irritative effects of epicutaneously applied haptens in mice Evidence that not the antigenic component but nonspecific proinflammatory effects of haptens determine the concentration-dependent elicitation of allergic contact dermatitis. J Clin Invest 98: 1158-1164, 1996

23. Olmos A, Giner RM, Recio MC, et al: Effects of plant alkylphenols on cytokine production, tyrosine nitration and inflammatory damage in the efferent phase of contact hypersensitivity. Br J Pharmacol 152: 366-373, 2007.

24. Haskó G, Mabley JG, Németh ZH, et al: Poly(ADP-ribose) polymerase is a regulator of chemokine production: relevance for the pathogenesis of shock and inflammation. Mol Med 8: 283-289, 2002 .
25. Soriano F, Virág L, Jagtap P, et al: Diabetic endothelial dysfunction: the role of poly(ADP-ribose) polymerase activation. Nat Med 7: 108-113, 2001

26. Bai P, Hegedus C, Szabó E, et al: Poly(ADP-ribose) polymerase mediates inflammation in a mouse model of contact hypersensitivity. J Invest Dermatol 129: 234-238, 2009.

27. de Murcia JM, Niedergang C, Trucco C, Ricoul M, Dutrillaux B, Mark M, Oliver FJ, Masson M, Dierich A, LeMeur M, Walztinger C, Chambon P, de Murcia G: Requirement of poly(ADP-ribose) polymerase in recovery from DNA damage in mice and in cells. Proc Natl Acad Sci USA. 94: 7303-7307, 1997.

28. Szklenar M, Kalkowski J, Stangl V, Lorenz M and Rühl R: Eicosanoids and docosanoids in plasma and aorta of healthy and atherosclerotic rabbits. J Vasc Res 50: 372-382, 2013.

29. Rühl R: Method to determine 4-oxo-retinoic acids, retinoic acids and retinol in serum and cell extracts by liquid chromatography/diode-array detection atmospheric pressure chemical ionisation tandem mass spectrometry. Rapid Commun Mass Spectrom 20: 2497-2504, 2006

30. Szabó E, Virág L, Bakondi E, et al: Peroxynitrite production, DNA breakage, and poly(ADP-ribose) polymerase activation in a mouse model of oxazolone-induced contact hypersensitivity. J Invest Dermatol 117: 74-80, 2001.

31. Elabdeen HR, Mustafa M, Szklenar M, et al: Ratio of pro-resolving and pro-inflammatory lipid mediator precursors as potential markers for aggressive periodontitis. PLoS One 8: e70838, 2013.

32. Lin Y, Tang X, Zhu Y, Shu T and Han X: Identification of PARP-1 as one of the transcription factors binding to the repressor element in the promoter region of COX-2. Arch Biochem Biophys 505: 123-129, 2011.

33. Hanhoff T, Lücke $C$ and Spener F: Insights into binding of fatty acids by fatty acid binding proteins. Mol Cell Biochem 239: 45-54, 2002.

34. Bai P and Cantó C: The role of PARP-1 and PARP-2 enzymes in metabolic regulation and disease. Cell Metab 16: 290-295, 2012.

35. Szántó M, Brunyánszki A, Márton J, et al: Deletion of PARP-2 induces hepatic cholesterol accumulation and decrease in HDL levels. Biochem Biophys Acta 1842: 594-602, 2014.

36. Tomobe YI, Morizawa K, Tsuchida M, et al: Dietary docosahexaenoic acid suppresses inflammation and immunoresponses in contact hypersensitivity reaction in mice. Lipids 35: 61-69, 2000.

37. Abba C, Mussa PP, Vercelli A and Raviri G: Essential fatty acids supplementation in different-stage atopic dogs fed on a controlled diet. J Anim Physiol Anim Nutr (Berl) 89: 203-207, 2005.

38. Rühl R, Koch C, Marosvölgyi T, et al: Fatty acid composition of serum lipid classes in mice following allergic sensitisation with or without dietary docosahexaenoic acid-enriched fish oil substitution. Br J Nutr 99: 1239-1246, 2008.

39. Virág L and Szabó C: The therapeutic potential of poly(ADP-ribose) polymerase inhibitors. Pharmacol Rev 54: 375-429, 2002.

40. Madsen P, Rasmussen HH, Leffers H, Honoré B and Celis JE: Molecular cloning and expression of a novel keratinocyte protein (psoriasis-associated fatty acid-binding protein [PA-FABP]) that is highly up-regulated in psoriatic skin and that shares similarity to fatty acid-binding proteins. J Invest Dermatol 99: 299-305, 1992. 